\section{Efeito do tempo de espera para radioterapia na sobrevida geral em cinco anos de mulheres com câncer do colo do útero, 1995-2010}

\author{
Effect of waiting time for radiotherapy on \\ five-year overall survival in women with \\ cervical cancer, 1995-2010
}

\author{
Efecto del tiempo de espera para radioterapia en \\ una sobrevida global en 5 años de mujeres con \\ cáncer del cuello uterino, 1995-2010
}

Maria Isabel do Nascimento 1

Gulnar Azevedo e Silva ${ }^{2}$

\section{Resumo}

\footnotetext{
${ }^{1}$ Instituto de Saúde Coletiva, Universidade Federal

Fluminense, Niterói, Brasil 2 Instituto de Medicina Social. Universidade do Estado do Rio de Janeiro, Rio de Janeiro, Brasil.

Correspondência

M. I. Nascimento

Departamento de

Epidemiologia e

Bioestatística, Instituto de

Saúde Coletiva, Universidade

Federal Fluminense.

Rua Marques do Paraná 303

3 o andar, Niterói, RJ

24033-900, Brasil.

ysamaria@uol.com.br
}

A sobrevida geral em cinco anos e os fatores associados ao óbito foram avaliados em uma coorte de 342 mulheres com câncer do colo uterino indicadas para radioterapia na Baixada Fluminense, Rio de Janeiro, Brasil. A sobrevida geral foi de $25,3 \%$, alcançando $60,8 \%$ para os casos até IIA. O ajustamento com uso do modelo de riscos proporcionais de Cox estendido mostrou risco de óbito aumentado para tumores IIBIIIB (HR = 1,89; IC95\%: 1,214; 2,957) e IVA-IVB (HR = 5,78; IC95\%: 2,973; 11,265). A captação por citologia (HR =0,58; IC95\%: 0,362; 0,961) e o encaminhamento direto para o serviço de radioterapia na Baixada Fluminese (HR = 0,60; IC95\%: 0,418; 0,875) foram os principais fatores protetores encontrados. O tempo de espera pela radioterapia ( $>60$ dias versus $\leq 60$ dias) foi não estatisticamente significativo, porém o retardo de quatro dias piorou os resultados (HR = 1,70; IC95\%: 1,153; 2,513). O limite de 60 dias para iniciar a radioterapia deve ser respeitado com rigor, pois, a partir de 64 dias, o retardo mostrou associação significativa entre todos os pontos de corte de tempo de espera analisados e o risco de morte em cinco anos.

Neoplasias do Colo do Útero; Radioterapia; Listas de Espera; Prognóstico; Análise de Sobrevida 


\section{Introdução}

O termo "tempo de espera" se refere à extensão de tempo que as pessoas aguardam para obterem os serviços de saúde de que necessitam. É uma medida utilizada em alguns países, como um indicador de desempenho de serviços de saúde na resolução de problemas considerados prioritários, incluindo o acesso ao tratamento do câncer 1,2

No Canadá, a mensuração do tempo de espera começa no momento em que o tratamento do câncer é requisitado, ou seja, no momento em que o paciente é considerado pronto para tratar e já tenha combinado com o médico o tipo de serviço que precisa utilizar, sendo estabelecido o prazo de 28 dias para o tratamento efetivamente começar 1 . No Reino Unido, o Department of Health determinou que o tratamento de qualquer tipo de câncer tivesse início dentro do prazo de 62 dias, a contar da referência médica na atenção primária, e que o tempo máximo aceitável da data do diagnóstico até a data do primeiro tratamento definitivo não ultrapassasse 31 dias ${ }^{3}$. O prazo de quatro semanas contado da data da primeira consulta com o oncologista especializado em radioterapia até a primeira sessão do tratamento foi estabelecido na Nova Zelândia 2 .

No contexto do tratamento do câncer, é importante assinalar que a radioterapia é uma alternativa adequada a todas as fases de tratamento de quase todos os tipos de tumores malignos, atingindo a taxa de utilização de 52\% (IC95\%: $51,7 ; 53,1)$ na modalidade de irradiação externa 4. Especificamente para os casos originados no colo do útero, a taxa de utilização varia de $54,3 \%$ a $67,9 \%$, com proporção de uso de $10,6 \%$ no estádio IA, $74,9 \%$ no estádio IB, chegando a $100 \%$ nos estádios II e III e 97,2\% no estádio IV 5. Nos Estados Unidos, a análise de 3.116 mulheres selecionadas junto ao Surveillance, Epidemiology, and End Results (SEER) mostrou um aumento no uso de radioterapia externa de $85 \%$ para $94 \%$ dos casos diagnosticados nos estágios II a IVA, entre 1997 e 2001 6. Segundo os autores, esse aumento possivelmente ocorreu em consequência de mudança no padrão terapêutico, com maior aplicação dessa terapia na malignidade cervical intermediária ou localmente avançada.

No Brasil, o câncer do colo do útero é um dos mais frequentes entre as mulheres, esperandose, para o ano 2014, a ocorrência de 15.590 casos novos, estimativa válida também para 2015 ? Apesar de a organização da atenção dar grande prioridade às ações de rastreamento da doença, mais de $75 \%$ dos casos atendidos no Instituto Nacional de Câncer José Alencar Gomes da
Silva (INCA) entre 1999 e 2004 encontravam-se em estágios II ou maior, implicando utilização de radioterapia em $69,8 \%$ dos casos, excluindo o estádio IV 8.

No Brasil, a extensão do tempo de espera para o tratamento do câncer foi determinada por lei em 22 de novembro de 2012, tendo sido estabelecido que todo paciente com câncer tenha o primeiro tratamento iniciado dentro do prazo de 60 dias a contar da data de registro do diagnóstico da doença no prontuário do paciente 9 Diante da crescente demanda pela radioterapia observada nos últimos anos e do grande interesse das autoridades governamentais em melhorar os resultados em câncer, o presente estudo teve como objetivos estimar a sobrevida geral em cinco anos e avaliar o efeito do tempo de espera para iniciar o tratamento radioterápico na sobrevida em cinco anos, em mulheres com câncer do colo do útero indicadas para radioterapia exclusiva na Baixada Fluminense, Rio de Janeiro, período 1995-2010.

\section{Métodos}

Este estudo tem delineamento de coorte retrospectivo e foi desenvolvido com dados de pacientes com câncer do colo do útero diagnosticadas em um hospital de ensino, referência para urgência e emergência do Sistema Único de Saúde (SUS), na região da Baixada Fluminense. O hospital abriga um polo secundário de colposcopia, habilitado para tratamento das lesões precursoras do câncer do colo do útero. Os casos invasivos são encaminhados para tratamento definitivo em unidades da rede de atenção oncológica da capital e/ou para um serviço isolado de radioterapia e quimioterapia prestador de serviços para o SUS, localizado na Baixada Fluminense.

\section{População de estudo}

A identificação dos casos foi feita por consulta aos arquivos dos setores de ginecologia, anatomia patológica e patologia cervical e colposcopia acessados no hospital de ensino. Foi identificado um total de 667 pacientes com câncer do colo do útero, livres de tratamento, atendidas no hospital de ensino entre outubro de 1995 e agosto de 2010. Houve casos de óbito antes do tratamento (51 casos), de tratamento primário por cirurgia (109 casos) e de insuficiência de dados essenciais para a identificação (26 casos), chegando-se ao total de 481 casos elegíveis. Foram excluídas oito mulheres que recusaram o tratamento, três que tiveram a radioterapia contraindicada e 109 que foram tratadas em unidades oncológicas da ca- 
pital do estado. Assim, a avaliação do tempo de espera para radioterapia na sobrevida em cinco anos foi conduzida com uma coorte de 342 pacientes que tiveram o tratamento radioterápico efetuado na unidade isolada regional de radioterapia e quimioterapia, na Baixada Fluminense.

\section{Fontes de dados}

Além do hospital de ensino, foram consultados os bancos de dados do serviço isolado de radioterapia e quimioterapia, onde está também instalado um registro hospitalar de câncer, para complementação de informações a respeito do tratamento efetuado. A terceira etapa contemplou a busca manual dos óbitos na base de dados identificada do Sistema de Informações sobre Mortalidade (SIM) da Secretaria Estadual de Saúde do Rio de Janeiro, disponibilizada a partir de 1995. Por último, foi feita a busca ativa com contato com pacientes ou familiares, com o objetivo de obter informações importantes, mas ainda faltantes a respeito de status vital.

\section{Coleta de dados e variáveis do estudo}

A coleta de dados foi orientada por um formulário criado especificamente para o estudo, com blocos de: (i) identificação da paciente, que incluiu as características sociodemográficas (idade ao diagnóstico, naturalidade, município de residência, cor da pele, situação conjugal, paridade e escolaridade); (ii) antecedentes ginecológicos; (iii) dados do diagnóstico do câncer e dos encaminhamentos; (iv) dados do tratamento e do seguimento; (v) história de citologia e (vi) contatos de acompanhantes e familiares.

O desfecho avaliado foi o óbito por qualquer causa dentro de cinco anos a contar da data de início da radioterapia no serviço isolado.

A exposição principal foi o tempo de espera para iniciar a radioterapia, definido como o intervalo entre a data de registro do diagnóstico histopatológico do câncer no prontuário da paciente arquivado no hospital de ensino e a data da primeira sessão de radioterapia no serviço isolado. $\mathrm{O}$ interesse principal foi verificar o efeito do tempo de espera categorizado em $>60$ dias versus até 60 dias na sobrevida geral em cinco anos. Pontos de corte adicionais ( $>75$ dias versus até 75 dias; > 90 dias versus até 90 dias; > 120 dias versus até 120 dias) foram também avaliados.

O período de seguimento foi estimado para cinco anos, com início do tempo de sobrevida (t0) estabelecido a partir da data (mais antiga) da primeira sessão de radioterapia. Pacientes com perda de seguimento e que terminaram o seguimento de cinco anos vivas foram censuradas. $\mathrm{O}$ período de seguimento foi iniciado em 18 de janeiro de 1996 e encerrado em 31 de dezembro de 2014.

A partir da combinação do estadiamento do tumor, estabelecido segundo critérios da Federação Internacional de Ginecologia e Obstetrícia (FIGO) 10, e do efeito esperado da radioterapia na cura da doença, foi criada a variável expectativa de cura, categorizada em três níveis: (i) tumores até IIA - potencialmente curáveis tanto por cirurgia quanto por radioterapia; (ii) tumores IIB até IIIB - potencialmente curáveis por radioterapia exclusiva; (iii) tumores IVA e IVB - tratamento paliativo por radioterapia.

As outras características de interesse foram a morfologia do tumor (escamoso, adenocarcinoma, outros tipos), a presença de sintoma hemorrágico (sim/não), o modo de captação do caso (por sintomas, por sintomas e citologia, por citologia em pacientes assintomáticas), uso de quimioterapia (sim/não), história de tabagismo (sim/não), tipo de referência para tratamento (direto para a unidade isolada versus indireto, isto é, intermediado em centro oncológico da capital). As variáveis com proporção de dados perdidos superior a $10 \%$ foram descartadas da análise uni e multivariada de sobrevivência.

\section{Análise de dados}

A análise de sobrevida foi conduzida, primeiramente, pela avaliação exploratória com uso de gráficos de Kaplan-Meier. A seguir, modelos de riscos proporcionais de Cox estendido, foram usados para obter hazard ratios (HR) brutas e ajustadas. A significância estatística foi avaliada na etapa univariada e multivariada com o uso do teste qui-quadrado de razão de verossimilhança, sendo selecionadas variáveis com valores de $\mathrm{p} \leq$ 0,20 e $\leq 0,05$, respectivamente. Fatores considerados importantes na relação exposição-desfecho foram incluídos no modelo, independente do valor de p. Foram calculados intervalos de $95 \%$ de confiança (IC95\%).

A verificação de interação foi feita com o uso do teste qui-quadrado de razão de verossimilhança. O pressuposto de proporcionalidade de risco global e de cada variável do modelo foi avaliado pela análise de resíduos de Schoenfeld. A análise foi conduzida com o uso da plataforma R (The R Foundation for Statistical Computing, Viena, Áustria; http://www.r-project.org).

\section{Aspectos éticos}

O estudo seguiu os princípios da Declaração de Helsinki e foi aprovado pelo Comitê de Ética em Pesquisa do Hospital Geral de Nova Iguaçu, 
com parecer de número 259.780 de 26 de março de 2013.

\section{Resultados}

Foi analisado um total de 342 pacientes com câncer do colo do útero histologicamente diagnosticado e tratado por radioterapia entre 1995 e 2010. A média de idade foi 51,7 anos (DP $=13,8$ anos; variação: 25 a 90 anos) e mais da metade das mulheres $(51,3 \%)$ eram casadas. A maioria $(60,1 \%)$ nasceu no Estado do Rio de Janeiro, e du- as pacientes tinham nacionalidade portuguesa. O tipo morfológico mais frequente foi o escamoso $(90,9 \%)$, seguido de adenocarcinoma $(5,8 \%)$, adenoescamoso $(1,5 \%)$ e outros tipos $(1,8 \%)$. Predominaram os casos não elegíveis para tratamento cirúrgico, com a distribuição de 33 casos (9,6\%) no estádio I, 135 (39,5\%) no estádio II, 157 (45,9\%) no estádio III e 17 (5\%) no estádio IV. A distribuição das características das pacientes no momento do diagnóstico está apresentada na Tabela 1.

Tabela 1

Caracterização dos 342 casos de câncer do colo do útero com tratamento por radioterapia exclusiva em unidade isolada regional da Baixada Fluminense, Rio de Janeiro, Brasil, 1995-2010.

\begin{tabular}{|c|c|}
\hline Variável & n (\%) \\
\hline \multicolumn{2}{|l|}{ Faixa etária (anos) } \\
\hline $20-29$ & $14(4,1)$ \\
\hline $30-39$ & $51(14,9)$ \\
\hline $40-49$ & $100(29,2)$ \\
\hline $50-59$ & $87(25,4)$ \\
\hline $60-69$ & $45(13,2)$ \\
\hline $70-79$ & $36(10,5)$ \\
\hline 80 e mais & $9(2,6)$ \\
\hline \multicolumn{2}{|l|}{ Idade (anos) } \\
\hline 60 e mais & $90(26,3)$ \\
\hline Até 59 & $252(73,7)$ \\
\hline \multicolumn{2}{|l|}{ Tipo histológico } \\
\hline Escamoso & $311(90,9)$ \\
\hline Adenocarcinoma & $20(5,8)$ \\
\hline Outros tipos & $11(3,3)$ \\
\hline \multicolumn{2}{|l|}{ Expectativa de cura } \\
\hline Potencialmente curável por cirurgia ou radioterapia & $55(16,1)$ \\
\hline Potencialmente curável por radioterapia exclusiva & $270(78,9)$ \\
\hline Radioterapia paliativa & $17(5,0)$ \\
\hline \multicolumn{2}{|l|}{ Modo de captação do caso } \\
\hline Por sintomas & $231(67,5)$ \\
\hline Por sintomas e citologia & $65(19,0)$ \\
\hline Por citologia em mulheres assintomáticas & $46(13,5)$ \\
\hline \multicolumn{2}{|l|}{ Hemorragia presente } \\
\hline Sim & $275(80,4)$ \\
\hline Não & $67(19,6)$ \\
\hline \multicolumn{2}{|l|}{ História de tabagismo } \\
\hline Fumante & $86(25,1)$ \\
\hline Ex-fumante & $41(12,0)$ \\
\hline Nunca fumou & $157(46,0)$ \\
\hline Sem informação & $58(16,9)$ \\
\hline \multicolumn{2}{|l|}{ Cor da pele } \\
\hline Não branca & $207(60,5)$ \\
\hline Branca & $129(37,7)$ \\
\hline Sem informações & $6(1,8)$ \\
\hline
\end{tabular}

(continua) 
Tabela 1 (continuação)

\begin{tabular}{|c|c|}
\hline Variável & n (\%) \\
\hline \multicolumn{2}{|c|}{ Residência no município do serviço de radioterapia } \\
\hline Sim & $199(58,2)$ \\
\hline Não & $143(41,8)$ \\
\hline \multicolumn{2}{|l|}{ Quimioterapia complementar à radioterapia } \\
\hline Sim & $150(43,9)$ \\
\hline Não & $192(56,1)$ \\
\hline \multicolumn{2}{|c|}{ Referência direta para a unidade de radioterapia } \\
\hline $\operatorname{Sim}$ & $181(52,9)$ \\
\hline Não & $161(47,1)$ \\
\hline \multicolumn{2}{|l|}{ Situação conjugal } \\
\hline Não casada & $155(45,3)$ \\
\hline Casada & $163(47,7)$ \\
\hline Sem informação & $24(7,0)$ \\
\hline \multicolumn{2}{|l|}{ Naturalidade } \\
\hline Rio de Janeiro & $194(56,7)$ \\
\hline Outra UF & $129(37,7)$ \\
\hline Sem informação & $19(5,6)$ \\
\hline \multicolumn{2}{|l|}{ Escolaridade } \\
\hline Analfabeta & $80(23,4)$ \\
\hline Fundamental incompleto até 3 anos & $74(21,6)$ \\
\hline Fundamental incompleto de 4 a 8 anos & $110(32,2)$ \\
\hline Fundamental completo e maior & $30(8,8)$ \\
\hline Sem informação & $48(14,0)$ \\
\hline \multicolumn{2}{|l|}{ Paridade (filhos) } \\
\hline Até 2 & $65(19,0)$ \\
\hline $3-6$ & $173(50,6)$ \\
\hline 7 ou mais & $84(24,6)$ \\
\hline Sem informação & $20(5,8)$ \\
\hline
\end{tabular}

A sobrevida global em cinco anos para o conjunto de casos foi de 25,3\% (IC95\%: 15,5; 41,4), com tempo mediano de sobrevivência de 1.107 dias. A estratificação segundo estadiamento/tratamento mostrou grande variação, com sobrevida global de cinco anos de 60,8\% (IC95\%: 34,3; $100,0)$ para os casos considerados potencialmente curáveis tanto por cirurgia quanto por radioterapia (até IIA); de 21,7\% (IC95\%: 12,3; 38,2) para os tumores considerados potencialmente curáveis por radioterapia exclusiva (IIB a IIIB); e de $0 \%$ para os tumores indicados para tratamento radioterápico paliativo (IVA e IVB).

$\mathrm{Na}$ análise univariada, o tempo de espera para iniciar a radioterapia de até 60 dias se revelou fator estatisticamente significativo para o desfecho óbito em cinco anos (HR = 1,53; IC95\%: $1,111 ; 2,130)$. O mesmo ocorreu com os pontos de corte de 75 dias (HR = 1,75; IC95\%: 1,238; 2,478), 90 dias (HR = 1,59; IC95\%: 1,076; 2,366) e 120 dias (HR = 1,66; IC95\%: 1,006; 2,757).
As demais variáveis que se mostraram positivamente associadas ao óbito em cinco anos foram a expectativa de cura baseada no estadiamento/tratamento, presença de hemorragia, morfologia do tumor, cor da pele, quimioterapia complementar. Comparando com casos considerados potencialmente curáveis tanto por cirurgia quanto por radioterapia, aqueles classificados como potencialmente curáveis por radioterapia exclusiva - estádios IIB a IIIB (HR 2,10; IC95\%: 1,$370 ; 3,248$ ) - e os indicados para tratamento para radioterapia paliativa - estádios IVA e IVB (HR = 6,55; IC95\%: 3,433; 12,492) -, apresentaram pior prognóstico e maior risco de óbito em cinco anos. O modo de captação por citologia do caso assintomático também mostrou um efeito significativo na sobrevida geral em cinco anos, com redução de risco de óbito de $50 \%$ (HR = 0,51; IC95\%: 0,323; 0,833), comparado com captação por sintomas. A idade ao diagnóstico de 60 anos e mais, residência no mesmo município onde está 
localizado o serviço isolado e o encaminhamento direto ao serviço isolado sem intermediação na capital se revelaram fatores protetores para o risco de óbito em cinco anos. A Tabela 2 mostra a distribuição das principais características e a análise univariada implementada com o uso de modelagem de riscos proporcionais de Cox estendido.

Na análise multivariada, a variável expectativa de cura baseada no estadiamento/tratamento se mostrou forte fator prognóstico para óbito em cinco anos nos casos IIB a IIIB (HR = 1,89; IC95\%: 1,$214 ; 2,957$ ) e nos casos IVA e IVB (HR $=5,78$; IC95\%: 2,973; 11,265), comparados com os casos até IIA. As variáveis que apresentaram diminuição de risco estatisticamente significativo foram: modo de captação do caso por citologia (HR = 0,58; IC95\%: 0,362; 0,961), idade acima de 59 anos ao diagnóstico ( $\mathrm{HR}=0,64$; IC95\%: 0,459; 0,887), referência direta para o serviço isolado (HR = 0,60; IC95\%: 0,418; 0,875) e residência no mesmo município do serviço isolado ( $\mathrm{HR}=0,75$; IC95\%: 0,564; 0,996) (Modelo 1).

Um segundo modelo (Modelo 2) foi ajustado considerando-se o tempo de espera para radioterapia como principal exposição. Depois do ajustamento, o intervalo limitado em até 60 dias, comparado com mais de 60 dias, não se mostrou estatisticamente associado ao risco de morte em cinco anos (HR = 1,44; IC95\%: 0,982; 2,138). Os fatores expectativa de cura baseada no estadiamento/tratamento, modo de captação do caso, idade ao diagnóstico e residência no mesmo município do serviço isolado, previamente identificados no Modelo 1, permaneceram no Modelo 2 estatisticamente associados ao desfecho óbito. A Tabela 3 apresenta os Modelos Finais obtidos com a modelagem de riscos proporcionais de Cox estendido.

Antecipando que a verificação do tempo de espera de 60 dias não seria suficiente para fornecer evidência consistente do efeito da demora para iniciar o tratamento no desfecho óbito, modelos adicionais foram avaliados com pontos de corte do tempo de espera estabelecidos em 75 dias (HR = 1,96; IC95\%: 1,324; 2,924), em 90 dias (HR = 1,97; IC95\%: 1,264; 3,092) e em 120 dias (HR = 1,93;IC95\%: 1,109; 3,379). Todos os modelos evidenciaram aumento do risco de óbito em cinco anos nesta coorte de mulheres com câncer do colo do útero, ajustado pelas outras variáveis previamente identificadas no modelo com espera de até 60 dias.

Com base nesse achado, uma série de modelos foi avaliada com pontos de corte do tempo de espera subsequentemente estabelecidos a partir de 61 dias, até 74 dias. Essa etapa de verificação dos pontos de corte, um a um, mostrou que, a partir de 64 dias (HR = 1,70; IC95\%: 1,153; 2,513), havia aumento no risco de óbito em cinco anos estatisticamente significativo para todos os pontos de corte subsequentemente avaliados.

A verificação da proporcionalidade de risco das variáveis testadas nos modelos univariados e multivariados foi feita pela interpretação de gráficos de resíduos de Schoenfeld, com $\mathrm{p}$ valor dos modelos com $(p=0,661)$ e sem tempo de espera de 60 dias ( $\mathrm{p}=0,591)$, sugerindo que não há violação do pressuposto.

\section{Discussão}

O presente estudo mostrou que o limite de 60 dias para iniciar a radioterapia em mulheres com câncer do colo do útero deve ser respeitado com rigor extremo, pois o retardo para 64 dias aumentou significativamente o risco de morte em cinco anos, segundo todos os pontos de corte de tempo de espera subsequentemente analisados. Também foi possível demonstrar que, na presença de estadiamento mais avançado, há um aumento de mais de três vezes no risco de morte para as mulheres indicadas para radioterapia paliativa (IVA/IVB), quando comparadas com aquelas supostamente curáveis tanto por cirurgia quanto por radioterapia (até IIA).

A literatura é escassa com respeito a estudos que avaliem o efeito do tempo de espera na sobrevida de pacientes com câncer, sendo os resultados, algumas vezes, conflitantes. O enfoque em tumores de diferentes topografias, submetidos a diferentes esquemas terapêuticos, sob diferentes definições de tempo de espera e de desfechos acaba por dificultar a comparabilidade entre os estudos. No Canadá, pesquisas restritas à população de mulheres mostraram, por exemplo, que a demora acima de duas semanas está associada a pior prognóstico na sobrevida em cinco anos de mulheres com câncer do corpo do útero tratado por cirurgia 11. No Reino Unido, a avaliação de tumores originados na mama não evidenciou efeito estatisticamente significativo do tempo de espera de 25 a 38 dias e de 39 a 62 dias, no risco de morte em cinco anos de mulheres tratadas cirurgicamente, comparando-se com espera menor que 25 dias 12. Na Holanda, o risco de morte específico por câncer de mama tratado com cirurgia conservadora seguida de radioterapia diminuiu no grupo de mulheres que aguardaram mais tempo (57-112 dias), em comparação com o tercil de até 45 dias 13 . Nos Estados Unidos, o tempo de espera de 60 dias ou mais não afetou a sobrevida de mulheres com câncer de mama in situ ou localizado, porém, nos estádios mais avançados, o tempo de espera maior aumentou 
Tabela 2

Distribuição dos fatores selecionados e análise univariada de 342 casos de câncer do colo do útero com uso de modelo de riscos proporcionais de Cox estendido.

\begin{tabular}{|c|c|c|c|c|}
\hline \multirow[t]{2}{*}{ Variável } & \multirow{2}{*}{$\begin{array}{c}\text { Distribuiçãa } \\
\text { n (\%) }\end{array}$} & \multicolumn{3}{|c|}{ Análise univariada } \\
\hline & & HR bruta & IC95\% & Valor de $p$ \\
\hline \multicolumn{5}{|l|}{ Expectativa de cura } \\
\hline Estadiamento até IIA & $55(16,1)$ & 1,00 & & \\
\hline Estadiamento de IIB a IIIB & $270(78,9)$ & 2,10 & 1,$370 ; 3,248$ & $<0,001$ \\
\hline Estadiamento IVA e IVB & $17(5,0)$ & 6,55 & 3,$433 ; 12,492$ & $<0,001$ \\
\hline \multicolumn{5}{|l|}{ Modo de captação do caso } \\
\hline Por sintomas & $231(67,5)$ & 1,00 & & \\
\hline Por sintomas e citologia & $65(19,0)$ & 1,03 & 0,$728 ; 1,465$ & 0,855 \\
\hline Por citologia em assintomáticas & $46(13,5)$ & 0,51 & 0,$323 ; 0,833$ & 0,006 \\
\hline \multicolumn{5}{|l|}{ Hemorragia vaginal } \\
\hline Presente & $275(80,4)$ & 1,58 & 1,$083 ; 2,320$ & 0,018 \\
\hline Ausente & $67(19,6)$ & & & \\
\hline \multicolumn{5}{|l|}{ Morfologia do tumor } \\
\hline Escamoso & $311(91,0)$ & 1,00 & & \\
\hline Adenocarcinoma & $20(5,8)$ & 1,72 & 1,$007 ; 2,985$ & 0,047 \\
\hline Outros tipos & $11(3,2)$ & 1,57 & 0,$805 ; 3,088$ & 0,185 \\
\hline \multicolumn{5}{|l|}{ Cor da pele * } \\
\hline Não branca & $207(61,6)$ & 1,43 & 1,$074 ; 1,902$ & 0,014 \\
\hline Branca & $129(38,4)$ & & & \\
\hline \multicolumn{5}{|l|}{ Idade ao diagnóstico (anos) } \\
\hline 60 e mais & $90(26,3)$ & 0,71 & 0,$520 ; 0,989$ & 0,042 \\
\hline Até 59 anos & $252(73,7)$ & & & \\
\hline \multicolumn{5}{|c|}{ Residência no município do serviço de radioterapia } \\
\hline Sim & $199(58,2)$ & 0,75 & 0,$576 ; 0,998$ & 0,048 \\
\hline Não & $143(41,8)$ & & & \\
\hline \multicolumn{5}{|l|}{ Quimioterapia complementar } \\
\hline Sim & $150(43,9)$ & 1,49 & 1,$062 ; 2,091$ & 0,021 \\
\hline Não & $192(56,1)$ & & & \\
\hline \multicolumn{5}{|l|}{ Situação conjugal * } \\
\hline Não casada & $163(51,3)$ & 1,17 & 0,$886 ; 1,550$ & 0,265 \\
\hline Casada & $153(48,7)$ & & & \\
\hline \multicolumn{5}{|c|}{ Referência direta para unidade de radioterapia } \\
\hline Sim & $181(52,9)$ & 0,59 & 0,$412 ; 0,858$ & 0,005 \\
\hline Não & $161(47,1)$ & & & \\
\hline \multicolumn{5}{|l|}{ Paridade (filhos) * } \\
\hline Até 2 & $65(20,2)$ & 1,00 & & \\
\hline $3-6$ & $173(53,7)$ & 1,06 & 0,$739 ; 1,530$ & 0,737 \\
\hline 7 ou mais & $84(26,1)$ & 0,91 & 0,$594 ; 1,416$ & 0,698 \\
\hline \multicolumn{5}{|l|}{ Naturalidade * } \\
\hline Do Rio de Janeiro & $194(60,1)$ & 0,95 & 0,$716 ; 1,263$ & 0,728 \\
\hline Outra Unidade da Federação & $129(39,9)$ & & & \\
\hline \multicolumn{5}{|c|}{ Tempo de espera para radioterapia (dias) } \\
\hline Acima de 60 & $95(27,8)$ & 1,53 & 1,$111 ; 2,130$ & 0,009 \\
\hline Até 60 & $247(72,2)$ & & & \\
\hline
\end{tabular}

HR: hazard ratio; IC95\%: intervalo de 95\% de confiança.

* Variáveis com menos de $10 \%$ de informações perdidas. 
Tabela 3

Modelo Final 1 não ajustado por tempo de espera e Modelo Final 2 ajustado por tempo de espera para radioterapia, obtidos com o uso de modelo de riscos proporcionais de Cox estendido.

\begin{tabular}{|c|c|c|c|c|}
\hline \multirow[t]{2}{*}{ Variável } & \multicolumn{2}{|c|}{ Modelo 1 não ajustado por espera pela radioterapia } & \multicolumn{2}{|c|}{ Modelo 2 ajustado por espera pela radioterapia } \\
\hline & HR ajustada & IC95\% & HR ajustada & IC95\% \\
\hline \multicolumn{5}{|l|}{$\begin{array}{l}\text { Expectativa de cura baseada no } \\
\text { tratamento/estádio }\end{array}$} \\
\hline Estadiamento até IIA & 1,00 & & 1,00 & \\
\hline Estadiamento de IIB a IIIB & 1,89 & 1,$214 ; 2,957$ & 1,87 & 1,$202 ; 2,921$ \\
\hline Estadiamento IVA e IVB & 5,78 & 2,$973 ; 11,265$ & 5,39 & 2,$763 ; 10,528$ \\
\hline \multicolumn{5}{|l|}{ Modo de captação do caso } \\
\hline Por sintomas & 1,00 & & 1,00 & \\
\hline Por sintomas e citologia & 1,12 & 0,$785 ; 1,601$ & 1,10 & 0,$769 ; 1,570$ \\
\hline Por citologia em assintomáticas & 0,58 & 0,$362 ; 0,961$ & 0,54 & 0,$329 ; 0,888$ \\
\hline \multicolumn{5}{|l|}{ Referência direta para radioterapia } \\
\hline $\operatorname{sim}$ & 0,60 & 0,$418 ; 0,875$ & 0,73 & 0,$482 ; 1,129$ \\
\hline \multicolumn{5}{|l|}{ Idade ao diagnóstico (anos) } \\
\hline Acima de 60 & 0,64 & 0,$459 ; 0,887$ & 0,62 & 0,$447 ; 0,865$ \\
\hline \multicolumn{5}{|c|}{ Residência no município da radioterapia } \\
\hline $\operatorname{Sim}$ & 0,75 & 0,$564 ; 0,996$ & 0,75 & 0,$560 ; 0,989$ \\
\hline \multicolumn{5}{|c|}{ Tempo de espera para radioterapia (dias) } \\
\hline Acima de 60 & - & Não testado & 1,44 & 0,$982 ; 2,138$ \\
\hline \multicolumn{5}{|l|}{ Teste de proporcionalidade de risco } \\
\hline global & & $p=0,591$ & & $p=0,661$ \\
\hline
\end{tabular}

HR: hazard ratio; IC95\%: intervalo de 95\% de confiança.

em $66 \%$ e em $85 \%$, respectivamente, o risco de morte por todas as causas e específico por câncer de mama, comparando-se com espera inferior a 60 dias 14

Ao contrário do câncer de mama, o efeito do tempo de espera para o tratamento do câncer do colo do útero tem sido menos frequentemente avaliado. No Canadá, a avaliação de 195 casos de câncer do colo do útero nos estádios IB a IVA tratados entre 1991 e 2001 evidenciou taxa de sobrevida geral em cinco anos de $53 \% 15$. Os autores verificaram aumento de $100 \%$ no risco de óbito em caso de atraso de cinco semanas ou mais para iniciar a radioterapia, com intervalo de tempo estipulado a partir da data da biópsia. No Japão, a estimativa de sobrevida geral em cinco anos foi alta, acima de $90 \%$ para casos com doença inicial (IA a IIA) e tempo de espera para cirurgia menor que 50 dias, em comparação com tempo de espera de 50 dias ou mais 16. Em Israel, a análise de 321 pacientes com doença maligna do colo do útero diagnosticada entre 1999 e 2010 não mostrou diferença na sobrevida em três anos, segundo o tempo de espera para iniciar o tratamento estratificado em até 30 dias, acima de 30 até 45 dias e acima de 45 dias 17.
Nesta investigação, outro resultado que merece destaque foi o alcançado pela análise da citologia, mostrando tratar-se de procedimento que efetivamente contribuiu para a obtenção de melhores resultados. Esse achado evidencia área que merece grandes investimentos na região da Baixada Fluminense, em face, principalmente, da proporção de pacientes assintomáticas captadas exclusivamente pela presença de alterações citológicas (13,5\%). Embora o percentual pareça baixo, sugerindo que pode melhorar ainda mais, ele é similar ao encontrado na Inglaterra em 2007 , onde $14 \%$ dos casos de câncer cervical foram captados por rastreamento citológico 18. Assim, além do grande papel na detecção de lesões precursoras e na prevenção do câncer do colo, seu uso ajudou a reduzir o risco de morte em $40 \%$ entre pacientes assintomáticas, quando comparadas com aquelas cujo atendimento foi motivado por alguma manifestação clínica.

A maior parte da coorte $(90,4 \%)$ tinha extensão de doença no estádio II ou maior, proporção superior à encontrada no Nepal $(86,4 \%)$ 19, em 2010, e mais do que o dobro da relatada na Dinamarca (42\%) 20, entre 2005 e 2010. O predomínio de diagnóstico tardio permite predizer que, pos- 
sivelmente, muitas mulheres vivem com a doença durante um longo tempo até obter diagnóstico e tratamento, dando uma ideia das condições de acesso aos serviços de saúde e do quanto se tem que avançar nas ações de controle do câncer do colo do útero na região da Baixada Fluminense.

A resposta ao tratamento na presença de doença avançada tem se mostrado pior, com repercussão no encurtamento da sobrevida em outras coortes no país 8,21 e no mundo 22,23. Mesmo se tratando de pacientes elegíveis unicamente para radioterapia, os atuais resultados alertaram para uma situação preocupante, visto que a sobrevida estimada nos cinco anos foi de apenas $25,3 \%$, considerando-se a coorte como um todo. Esse achado pode ser atribuído à grande proporção de casos com doença localmente avançada, condição previamente encontrada no INCA 8 , onde a sobrevida foi de $48 \%$ na coorte que contava também com pacientes tratadas por cirurgia. No presente estudo, a análise estratificada por subgrupos de estadiamento/tratamento evidenciou a taxa de $60 \%$ na estimativa de sobrevida geral em cinco anos para os casos em estágios mais precoces, elegíveis tanto para cirurgia quanto para radioterapia, reforçando a ideia do prejuízo imposto às mulheres pela defasagem de tempo até o diagnóstico.

O retardo no diagnóstico do câncer decorre de uma sequência de fatos que começa pelo desconhecimento por parte dos pacientes sobre os sinais e sintomas de suspeição da doença, o que não lhes inspira a buscar ajuda de profissionais de saúde. O médico também responde por parte da demora quando desconhece quadros sintomáticos causados pela doença, postergando a investigação e o encaminhamento para especialistas. Ao sistema, cabe responsabilidades por esperas desnecessárias na implementação de investigações, bem como pela extensão do intervalo entre a decisão de tratar e o efetivo início do tratamento 24 . Modelos matemáticos mostram que aspectos inerentes à radiossensibilidade e a taxa de repopularização tumoral também contribuem para resultados mais pobres. A redução na probabilidade de controle de tumores escamosos do colo do útero considerados mais heterogêneos e agressivos variou de 1,9\% a 3,9\% na presença de demora de 50 dias e de 100 dias para iniciar a radioterapia, respectivamente 25.

Este estudo tem limitações em decorrência, principalmente, de sua natureza retrospectiva. Primeiramente, 51 pacientes morreram na fase diagnóstica e de estadiamento antes de preencherem todos os critérios para encaminhamento para radioterapia, caracterizando estudo conduzido com dados prevalentes. Para lidar com essa situação e evitar o viés de sobrevida mais longa, a estratégia foi usar o tempo calendário e empregar o modelo de riscos proporcionais de Cox estendido 26. Ademais, as informações sobre hábito de fumar e escolaridade estavam incompletas em percentual acima de $10 \%$, por isso não foram incluídas na análise. Apesar da importância do tabagismo como fator de risco para vários tipos de câncer, seu efeito nos resultados de tratamentos do câncer cervical não tem sido confirmado 17,27. Possivelmente, os resultados do presente estudo se repetiriam na presença de informação mais completa sobre tabagismo. Com relação à escolaridade, há evidência, no Brasil, de que se trata de fator que influencia pouco na sobrevida em cinco anos de mulheres com câncer do colo do útero ${ }^{8,21}$. Além disso, o fato de todas as mulheres residirem na Baixada Fluminense, região onde predominam Índices de Desenvolvimento Humano Municipal (IDHM) 28 de até 0,761, permite pressupor que se trata de coorte com perfil socioeconômico homogêneo, o que pode ajudar a compensar a ausência de informações sobre a escolaridade. Outra circunstância que poderia ter afetado os resultados da coorte estudada foram as perdas de seguimento. Ainda que estas tenham atingido o percentual de $19 \%$, boa parte das mulheres que deixaram de ser seguidas $(10,8 \%)$ permaneceu em acompanhamento por mais de 24 meses, minimizando o possível efeito na estimativa de sobrevida. Não foram observadas diferenças estatisticamente significativas na comparação dos grupos sem e com seguimento completo no que diz respeito a algumas características selecionadas, como média de idade (53,3 anos versus 51,3 anos; $p=0,308$ ), naturalidade do Rio de Janeiro (58,3\% versus $60,5 \%$; $\mathrm{p}=0,762)$, sem companheiro $(43,6 \%$ versus $49,8 \%$, $\mathrm{p}=0,405)$, cor da pele não branca $(60 \%$ versus $62 \%, p=0,767$ ) e residência no mesmo município do centro de radioterapia $(66,2 \%$ versus $56,2 \%$, $\mathrm{p}=0,136$ ). Por fim, embora se contasse com um grupo de 12 mulheres que iniciaram a radioterapia no ano 2010, o seguimento previsto de cinco anos foi encerrado ao final de 2014. Além de a análise parcial com exclusão destes casos não ter mostrado influência nos resultados encontrados, já se havia constatado óbito de nove das 12 mulheres e perda de seguimento de outras três.

Em vez de se arbitrar a definição e ponto de corte do tempo de espera mais conveniente, este estudo teve, entre as vantagens, a adoção de parâmetros conceituais estabelecidos pela Lei no 12.732 de 22 de novembro de 2012, a qual regulamenta o primeiro tratamento do câncer no país ${ }^{9}$, favorecendo a interpretação dos resultados e futuras comparações. O fato de todos os casos residirem na Baixada Fluminense, região que tem cinco (Belford Roxo, Japeri, Nova Iguaçu, 
Queimados e Magé) dos seus 12 municípios com índice de vulneráveis à pobreza ${ }^{28}$ superior a $30 \%$, pode ter ajudado a minimizar os possíveis efeitos de viés de seleção, uma vez que a pobreza é uma condição relacionada à malignidade cervical 29. O estudo apenas incluiu tumores primários do colo do útero, com indicação de radioterapia exclusiva, formando coorte bastante homogênea com respeito também às características da doença, podendo facilitar, assim, o entendimento de como está ocorrendo o acesso ao diagnóstico e ao tratamento do câncer do colo do útero fora da capital do Estado do Rio de Janeiro.

Os dados permitiram concluir que a espera de até 60 dias avaliada de modo individual aumentou o risco de morte, reforçando a ideia de que, perante o diagnóstico do câncer, o tratamento deve ser iniciado o mais precocemente possível, de preferência antes de 60 dias. Depois do ajustamento, o efeito se manteve, mas não repercutiu em aumento efetivo no risco de morte. Entretanto, a postergação desse prazo por mais quatro dias, novamente, piorou os resultados com aumento de $70 \%$ no risco de óbito em cinco anos (ajustado pelos outros fatores do modelo). Tendo em vista as especificidades da população estudada, os achados deste estudo devem ser interpretados com cautela.

Concluindo, o estadiamento mais avançado do tumor, o encaminhamento direto para o serviço isolado, a residência no mesmo município do serviço isolado e a captação por citologia de casos assintomáticos são os principais fatores prognósticos que influenciaram a sobrevida em cinco anos. Independente da legislação vigente no país, no caso do câncer do colo do útero com indicação de tratamento exclusivo por radioterapia, o estudo sugere que esforços de todas as partes envolvidas no controle da doença devem ser direcionados não só para cumprir adequadamente as etapas pré-tratamento e iniciar a radioterapia no prazo máximo de 60 dias, como também para ampliar o acesso das mulheres à citologia.

\section{Resumen}

La sobrevida global en 5 años y los factores asociados a la muerte fueron evaluados en una cohorte de 342 mujeres con cáncer de cuello uterino, elegidas para radioterapia en la Baixada Fluminense, Rio de Janeiro, Brasil. La sobrevida global fue 25,3\%, alcanzando $60,8 \%$ en estadios clínicos hasta IIA. El ajuste obtenido con el modelo de riesgo proporcional de Cox extendido expuso un riesgo de muerte más elevado en mujeres con estadios IIB-IIIB ( $R R=1,89$; IC95\%: 1,214; 2,957) y IVA-IVB (RR = 5,78; IC95\%: 2,973; 11,265). La citología ( $R R=0,58$; IC95\%: 0,362; 0,961) y el envío directo hacia el servicio oncológico en Baixada Fluminense $(R R=$
0,60; IC95\%:0,418;0,875) fueron los factores protectores encontrados. El tiempo de espera (> 60 días versus $\leq 60$ días) no resultó en una diferencia significativa, pero el retraso de 4 días empeoró los resultados $(R R=1,70$; IC95\%: 1,153; 2,513). El límite de 60 días para empezar la radioterapia debe ser respetado con rigor, puesto que a partir de 64 dias, la tardanza mostró asociación significativa entre todos los puntos de corte de tiempo de espera evaluado y el riesgo de morir en 5 años.

Neoplasias del Cuello Uterino; Radioterapia; Listas de Espera; Pronóstico; Análisis de Supervivencia 


\section{Colaboradores}

M. I. Nascimento e G. Azevedo e Silva trabalharam conjuntamente na concepção do estudo, interpretação dos resultados e redação do artigo. M. I. Nascimento foi responsável pela coleta e análise dos dados. G. Azevedo e Silva realizou a revisão crítica do conteúdo e aprovou a versão final.

\section{Agradecimentos}

As autoras agradecem ao CNPq pela concessão de Bolsa de Pesquisador Júnior, percebida por M. I. Nascimento, sob supervisão de G. Azevedo e Silva, processo no 150063/2013-0.

\section{Referências}

1. Canadian Institute for Health Information. Wait time information in priority areas: definitions. http://www.cihi.ca/CIHI-ext-portal/internet (acessado em 20/Set/2014).

2. Ministry of Health. Targeting waiting times. Wellington: Ministry of Health; 2013.

3. Department of Health. The NHS Cancer Plan: a plan for investment, a plan for reform. London: Department of Health; 2000.

4. Delaney G, Jacob S, Featherstone C, Barton M. The role of radiotherapy in cancer treatment: estimating optimal utilization from a review of evidence-based clinical guidelines. Cancer 2005; 104: 1129-37.

5. Usmani N, Foroudi F, Du J, Zakos C, Campbell H, Bryson P, et al. An evidence-based estimate of the appropriate rate of utilization of radiotherapy for cancer of the cervix. Int J Radiat Oncol Biol Phys 2005; 63:812-27.

6. Trimble EL, Harlan LC, Gius D, Stevens J, Schwartz SM. Patterns of care for women with cervical cancer in the United States. Cancer 2008; 113:743-9.

7. Coordenação de Prevenção e Vigilância, Instituto Nacional de Câncer José Alencar Gomes da Silva. Estimativa 2014: incidência de câncer no Brasil. Rio de Janeiro: Instituto Nacional de Câncer José Alencar Gomes da Silva; 2014.

8. Carmo CC, Luiz RR. Survival of a cohort of women with cervical cancer diagnosed in a Brazilian cancer center. Rev Saúde Pública 2011; 45:661-7.

9. Brasil. Lei no 12.732, de 22 de novembro de 2012. Dispõe sobre o primeiro tratamento de paciente com neoplasia maligna comprovada e estabelece prazo para seu início. Diário Oficial da União 2012; 23 nov.

10. Benedet JL, Bender H, Jones 3rd H, Ngan HY, Pecorelli S. FIGO staging classifications and clinical practice guidelines in the management of gynecologic cancers. FIGO Committee on Gynecologic Oncology. Int J Gynaecol Obstet 2000; 70: 209-62.

11. Elit LM, O'Leary EM, Pond GR, Seow HY. Impact of wait times on survival for women with uterine cancer. J Clin Oncol 2014; 32:27-33.

12. Redaniel MT, Martin RM, Cawthorn S, Wade J, Jeffreys $M$. The association of waiting times from diagnosis to surgery with survival in women with localised breast cancer in England. Br J Cancer 2013; 109:42-9.

13. Jobsen JJ, van der Palen J, Baum M, Brinkhuis M, Struikmans H. Timing of radiotherapy in breastconserving therapy: a large prospective cohort study of node-negative breast cancer patients without adjuvant systemic therapy. Br J Cancer 2013; 108:820-5.

14. McLaughlin JM, Anderson RT, Ferketich AK, Seiber EE, Balkrishnan R, Paskett ED. Effect on survival of longer intervals between confirmed diagnosis and treatment initiation among low-income women with breast cancer. J Clin Oncol 2012; 30:4493-500.

15. Choan E, Dahrouge S, Samant R, Mirzaei A, Price J. Radical radiotherapy for cervix cancer: the effect of waiting time on outcome. Int J Radiat Oncol Biol Phys 2005; 61:1071-7. 
16. Umezu T, Shibata K, Kajiyama H, Yamamoto E, Mizuno M, Kikkawa F. Prognostic factors in satge IA-IIA cervical cancer patients treated surgically: does the waiting time to the operation affect survival? Arch Gynecol Obstet 2012; 285:493-7.

17. Perri T, Issakov G, Ben-Baruch G, Felder S, Beiner ME, Helpman L, et al. Effect of treatment delay on survival in patients with cervical cancer: a historical cohort study. Int J Gynecol Cancer 2014; 24:1326-32.

18. National Cancer Intelligent Network. Routes to diagnosis data briefing. http://www.ncin.org.uk/ publications/data_briefing/routes_to_diagnosis (acessado em 20/Nov/2012).

19. Gyenwali D, Khanal G, Paudel R, Amatya A, Pariyar J, Onta SR. Estimates of delays in diagnosis of cervical cancer in Nepal. BMC Womens Health 2014; $14: 29$.

20. Ibfelt EH, Kjaer SK, Hogdall C, Steding-Jessen M, Kjaer TK, Osler M, et al. Socioeconomic position and survival after cervical cancer: influence of cancer stage, comorbidity and smoking among Danish women diagnosed between 2005 and 2010. Br J Cancer 2013; 109:2489-95.

21. Mascarello KC, Zandonade E, Amorim MH. Survival analysis of women with cervical cancer treated at a referral hospital for oncology in Espírito Santo State, Brazil, 2000-2005. Cad Saúde Pública 2013; 29:823-31.
22. Kantelhardt EJ, Moelle U, Begoihn M, Addissie A, Trocchi P, Yonas B, et al. Cervical cancer in Ethiopia: survival of 1,059 patients who received oncologic therapy. Oncologist 2014; 19:727-34.

23. Wabinga $\mathrm{H}$, Ramanakumar AV, Banura C, Luwaga A, Nambooze S, Parkin DM. Survival of cervix cancer patients in Kampala, Uganda: 1995-1997. Br J Cancer 2003; 89:65-9.

24. Foot C, Harrison T. Cancer research UK. How to improve cancer survival: explaining England's relatively poor rates. London: The King's Fund; 2011.

25. Wyatt RM. The effects of delays in radiotherapy treatment on tumour control. Phys Med Biol 2003; 48:139-55.

26. Carvalho MS, Andreozzi VL, Codeço CT, Campos DP, Barbosa MTS, Shimakura SE. Análise de Sobrevivência: teoria e aplicações em saúde. $2^{\text {a }}$ Ed. Rio de Janeiro: Editora Fiocruz; 2011.

27. Fyles A, Voduc D, Syed A, Milosevic M, Pintilie M, Hill R. The effect of smoking on tumour oxygenation and treatment outcome in cervical cancer. Clin Oncol 2002; 14:442-6.

28. Programa das Nações Unidas para o Desenvolvimento. Atlas do desenvolvimento humano no Brasil. http://www.atlasbrasil.org.br (acessado em 23/ Abr/2015).

29. Lima CA, Palmeira JAV, Cipolotti R. Fatores associados ao câncer do colo uterino em Propriá, Sergipe, Brasil. Cad Saúde Pública 2006; 22:2151-6.

Recebido em 14/Jan/2015

Versão final reapresentada em 24/Abr/2015

Aprovado em 21/Mai/2015 